\title{
Criminologie
}

\section{Facteurs associés à la décision d'entreprendre des poursuites criminelles à la suite d'un signalement pour abus sexuel ou physique envers un enfant}

\section{Marc Tourigny et Nico Trocmé}

Volume 33, numéro 2, automne 2000

Problèmes sociaux et système pénal

URI : https://id.erudit.org/iderudit/004750ar

DOI : https://doi.org/10.7202/004750ar

Aller au sommaire du numéro

Éditeur(s)

Les Presses de l'Université de Montréal

ISSN

0316-0041 (imprimé)

1492-1367 (numérique)

Découvrir la revue

Citer cet article

Tourigny, M. \& Trocmé, N. (2000). Facteurs associés à la décision d'entreprendre des poursuites criminelles à la suite d'un signalement pour abus sexuel ou physique envers un enfant. Criminologie, 33(2), 7-30.

https://doi.org/10.7202/004750ar
Résumé de l'article

L'Étude ontarienne sur l'incidence des mauvais traitements (ÉOI) est une des premières études examinant en détails les caractéristiques des cas d'abus physique $(\mathrm{N}=1003)$ et d'abus sexuel $(\mathrm{N}=577)$ signalés aux services de protection de l'enfance. Les abus sexuels se caractérisent par le fait que $16 \%$ concernent des relations sexuelles ou des tentatives et $56 \%$ des attouchements sexuels. Les principaux agresseurs présumés sont une figure paternelle ( $34 \%$ ), un membre de la famille autre que le parent $(24 \%)$ ou une connaissance de la famille $(23 \%)$. Près des deux tiers des situations d'abus physique concernent des problèmes de discipline ( $65 \%$ ), des blessures physiques sont notées dans $27 \%$ des cas, et les figures parentales représentent la très grande majorité des agresseurs présumés (92\%). Des poursuites criminelles ont été entamées dans $15 \%$ des cas d'abus sexuel et dans $5 \%$ des cas d'abus physique. Quatre facteurs se sont avérés associés aux décisions d'entamer des poursuites pénales dans les cas d'abus sexuel : la nature des abus sexuels (les cas de relations sexuelles ayant la plus grande probabilité de mener à des poursuites), la nature des séquelles, l'âge de l'enfant signalé (les poursuites étant plus probables pour les victimes plus âgées) et la source du signalement. Trois facteurs étaient associés aux décisions d'engager des poursuites pénales à la suite d'évaluations d'abus physique : la présence de blessures, la source du signalement et la région de la province où s'est déroulée l'enquête.
Ce document est protégé par la loi sur le droit d'auteur. L'utilisation des services d'Érudit (y compris la reproduction) est assujettie à sa politique d'utilisation que vous pouvez consulter en ligne.

https://apropos.erudit.org/fr/usagers/politique-dutilisation/ 


\title{
Facteurs associés à la décision d'entreprendre des poursuites criminelles à la suite d'un signalement pour abus sexuel ou physique envers un enfant
}

\author{
Nico Trocmé \\ Professeur \\ Faculté de service social \\ Université de Toronto \\ nico.trocme@utoronto.ca \\ Marc Tourigny \\ Professeur \\ Département de psychoéducation \\ et de psychologie \\ Université du Québec à Hull \\ Marc_Tourigny@uqah.uquebec.ca
}

RÉSUMÉ - L'Étude ontarienne sur l'incidence des mauvais traitements (ÉOI) est une des premières études examinant en détails les caractéristiques des cas d'abus physique $(\mathrm{N}=1003)$ et d'abus sexuel $(\mathrm{N}=577)$ signalés aux services de protection de l'enfance. Les abus sexuels se caractérisent par le fait que $16 \%$ concernent des relations sexuelles ou des tentatives et $56 \%$ des attouchements sexuels. Les principaux agresseurs présumés sont une figure paternelle $(34 \%)$, un membre de la famille autre que le parent (24\%) ou une connaissance de la famille $(23 \%)$. Près des deux tiers des situations d'abus physique concernent des problèmes de discipline (65\%), des blessures physiques sont notées dans $27 \%$ des cas, et les figures parentales représentent la très grande majorité des agresseurs présumés (92\%). Des poursuites criminelles ont été entamées dans $15 \%$ des cas d'abus sexuel et dans $5 \%$ des cas d'abus physique. Quatre facteurs se sont avérés associés aux décisions d'entamer des poursuites pénales dans les cas d'abus sexuel : la nature des abus sexuels (les cas de relations sexuelles ayant la plus grande probabilité de mener à des poursuites), la nature des séquelles, l'âge de l'enfant signalé (les poursuites étant plus probables pour les victimes plus âgées) et la 
source du signalement. Trois facteurs étaient associés aux décisions d'engager des poursuites pénales à la suite d'évaluations d'abus physique : la présence de blessures, la source du signalement et la région de la province où s'est déroulée l'enquête.

ABSTRACT - The Ontario Incidence Study of Reported Child Abuse and Neglect (OIS) is one of the first studies to examine in detail the characteristics of physical abuse $(\mathrm{N}=1003)$ and sexual abuse $(\mathrm{N}=577)$ cases reported to child protection authorities. Sixteen per cent of sexual abuse cases involved intercourse or attempted intercourse and $56 \%$ involved fondling. The alleged perpertrators were most often fathers or stepfathers $(34 \%)$, relatives $(24 \%)$ or family acquaintances $(23 \%)$. Sixty-five per cent of physical abuse cases involved corporal punishemnt or incidents arising from a disciplinary action, physical injuries were noted in $27 \%$ of cases, and parents were most often the alleged perpertrators (92\%). Criminal charges had been laid in 15\% of sexual abuse cases and in $5 \%$ of physical abuse cases. Four variables were significantly associated with the decision to lay charges in sexual abuse cases: the level of intrusiveness of the abuse (cases involving intercourse or attempted intercourse were more likely to lead to charges), the nature of documented harm, the age of the reported child (charges were laid more often in cases involving older children) and the source of the report. Three variables were significantly associated with the decision to lay charges in physical abuse cases: the presence of an injury, the source of the report, and the region of the province in which the investigation was conducted.

\section{Introduction}

La protection des enfants victimes de mauvais traitements parentaux nécessite l'établissement d'un équilibre précaire entre les offres d'aide et de soutien d'un côté, et les menaces de punition de l'autre. La différence entre ces deux approches va en s'accentuant. Alors que certains auteurs font appel au développement d'approches communautaires et à l'utilisation de techniques de résolution de conflits pour éviter le recours aux tribunaux (Palmer, 1989; Burford et Pennell, 1995; Barsky et Trocmé, 1997), d'autres maintiennent que les mauvais traitements infligés aux enfants doivent être traités avant tout comme un acte criminel et ne pas être réduits à un problème familial (Besharov, 1990; Wells, 1990; Lindsey et Regehr, 1993; Lindsey, 1994). Ces mouvements se traduisent par une division de plus en plus nette entre les interventions volontaires et les poursuites pénales. En Ontario, par exemple, on note qu'un nombre croissant de familles reçoivent des services des agences de protection sans que les enfants soient pris en charge, et que ces agences ont de moins en moins recours aux tribunaux de la jeunesse mais font de plus en plus souvent appel à l'intervention de la police (Trocmé, 1991). 
Au Québec, Roberge (1998) a suivi le cheminement de 6304 signalements reçus dans six centres jeunesse (CJ) entre le $1^{\mathrm{er}}$ avril 1992 et le 31 mars 1993, et ce, sur une période moyenne de trois à quatre ans. Les résultats ont montré que selon les CJ, de $39 \%$ à $68 \%$ des cas pris en charge pour abus sexuel sont judiciarisés au tribunal de la jeunesse; il s'agit de la forme de compromissions où le recours à ce type de judiciarisation est le plus fréquent. À l'inverse, les abus physiques représentent la forme de compromissions la moins souvent judiciarisée, avec des pourcentages variant de $0 \%$ à $34 \%$ selon le CJ. Nous n'avons toutefois pas de données québécoises sur le recours à la judiciarisation pour la cour criminelle ou pénale.

En pratique, chaque système d'intervention tend à imposer sa propre définition de la problématique - acte criminel ou problème social ainsi que ses propres règles et limites. Par exemple, les règles entourant la preuve et les témoignages sont plus rigoureuses dans le système criminel que dans le système de protection de l'enfance. L'équilibre entre ces deux systèmes est maintenu par le développement de protocoles d'intervention et d'évaluations visant à favoriser la collaboration entre la police et les agences de protection des enfants. Ces protocoles d'entente déterminent dans quelles circonstances les deux systèmes entreprennent des évaluations conjointes et comment ces équipes de policiers et d'intervenants sociaux collaborent. En principe, cette approche assure que les enfants maltraités reçoivent la protection des deux systèmes. Quand les circonstances et les témoignages le permettent, les enquêtes policières donnent lieu à des poursuites pénales, tout en maintenant, si nécessaire, un certain soutien par le biais des agences de protection. Quand les circonstances sont telles que des poursuites pénales ne peuvent pas être engagées, la protection peut être assumée par les agences de protection ${ }^{1}$.

Malheureusement, nous avons très peu de renseignements jusqu'à maintenant sur la nature exacte de cette collaboration. Quelques études concernant surtout des situations d'abus sexuel se sont penchées sur les caractéristiques des dossiers qui sont engagés dans un processus judiciaire, c'est-à-dire qui étaient susceptibles de se rendre jusqu'au procès. En ce qui

1. En Ontario, par exemple, avec la Loi des services pour les enfants et les familles, il suffit de démontrer qu'il y a prépondérance de preuves que l'enfant a été victime ou est à risque imminent d'être victime de mauvais traitements (Ontario, 1990). En revanche, le système de justice criminelle requiert que les témoignages ou preuves démontrent la culpabilité de l'agresseur hors de tout doute raisonnable. 
concerne la plainte policière, l'incapacité ou le non-désir de la victime (ou ses parents) à témoigner, de même que l'insuffisance de preuves sont les principaux facteurs influençant la poursuite des démarches judiciaires (Sas et al., 1991; Hornick et Bolitho, 1992 : voir Schmolka, 1992). D'autres caractéristiques des dossiers sont également susceptibles d'influencer les décisions prises à chacune des étapes du processus judiciaire. Les dossiers qui parcourent l'ensemble du processus judiciaire présentent souvent les caractéristiques suivantes : 1) il s'agit de situations d'abus sexuel plutôt que d'autres formes de mauvais traitements (Tjaden et Thoennes, 1992); 2) les enfants sont plus âgés (Cross et De Vos, 1990 : voir Cross et al., 1994; Sas et al., 1991; Whitcomb et al., 1991; Tjaden et Thoennes, 1992; Gray, 1993; Cross et al., 1994); 3) les agresseurs sont sans lien de parenté (ou ont des liens moins intenses) avec l'enfant (Tjaden et Thoennes, 1992; Gray, 1993; Cross et al., 1994) et ne sont pas de race blanche (Tjaden et Thoennes, 1992); 4) les mauvais traitements se caractérisent par une grande fréquence, une longue durée et l'utilisation de la force physique (Cross et De Vos, 1990 : voir Cross et al., 1994; Whitcomb et al., 1991; Tjaden et Thoennes, 1992; Gray, 1993; Cross et al., 1994); 5) le dévoilement s'est fait auprès d'un ami ou d'une connaissance plutôt qu'auprès d'un membre de la famille ou d'un professionnel (Cross et al., 1994); 6) l'enquête a été réalisée d'abord par les policiers plutôt que par les agences de protection (Cross et al., 1994); 7) des preuves physiques ou la présence d'un témoin oculaire sont venues corroborer le témoignage de l'enfant (Gray, 1993; Cross et al., 1994); 8) l'enfant a bénéficié d'un plus grand soutien maternel et il montrait moins de détresse psychologique (Cross et al., 1994).

Malgré cette pauvreté de données empiriques, il est néanmoins possible d'identifier d'autres facteurs liés spécifiquement à la situation de mauvais traitements qui peuvent influencer les décisions d'entreprendre un recours judiciaire, et ce, à partir de la littérature clinique et des études examinant d'autres étapes décisionnelles, telles que le placement des enfants à l'extérieur de leur milieu familial et la confirmation des signalements (pour une revue de ces études voir Trocmé et al., 1995b et Tourigny, 1999). Ces études suggèrent que les facteurs suivants pourraient influencer les décisions d'engager des poursuites pénales : l'âge des enfants et leur capacité à agir comme témoins, la présence de preuves médico-légales, la présence de témoins et la sévérité des mauvais traitements. Le rôle important que jouent ces différents facteurs dépend en partie du type d'abus dont il est question. Dans les cas d'abus physique, 
une plus grande importance est accordée aux preuves médico-légales. La présence d'une blessure est pratiquement nécessaire pour entamer des poursuites pénales. Les blessures sont bien plus rares dans les cas d'abus sexuel, pour lesquels la crédibilité de l'enfant et la présence de témoins deviennent importants (Gray, 1993; Lanning, 1996; Walsh, 1996).

Finalement, il existerait d'autres facteurs moins directement liés à la situation de mauvais traitements mais qui influenceraient également les processus décisionnels. La source du signalement s'avère, dans un bon nombre d'études, avoir une influence importante sur les interventions des services de protection de l'enfance (Groenveld et Giovannoni, 1977; Runyan et al., 1981; Miller et al., 1982; Giovannoni, 1989; Lindsey, 1991). Par exemple, ces études ont toutes trouvé que les cas signalés par la police étaient traités de manière plus sérieuse que les cas signalés par d'autres sources de signalement. L'expérience de l'intervenant réalisant l'évaluation et des facteurs liés à l'agence de protection de l'enfance en particulier l'emplacement géographique de l'agence - s'avèrent, d'après certaines études, avoir également une influence sur les décisions prises (Runyan et al., 1981; Wolock, 1982; Trocmé et al., 1995b). Finalement, plusieurs études ont révélé que les facteurs de race et de classe sociale semblent aussi influencer les décisions d'intervention (Runyan $e t$ al., 1981; Hampton, 1987; Lindsey, 1991).

En somme, on peut distinguer deux catégories de facteurs pouvant influencer les décisions d'entamer des poursuites criminelles : d'une part, les facteurs intrinsèques qui sont directement liés à la situation de mauvais traitements et, d'autre part, les facteurs extrinsèques, tels que la source du signalement, l'emplacement géographique, ainsi que la race et la classe sociale de la famille signalée. L'objectif de cet article est donc d'examiner de manière empirique le rôle joué par ces facteurs quant aux décisions d'engager des poursuites pénales dans le contexte d'évaluations de signalements d'abus physique ou sexuel effectués aux agences ontariennes de protection de l'enfance. Un second objectif est de pouvoir décrire la nature des abus physiques et sexuels signalés, de même que les caractéristiques des enfants, des familles et de certaines interventions sociales et judiciaires réalisées durant l'évaluation du signalement. 


\section{Méthodologie}

Devis

L'étude porte sur l'analyse d'un sous-ensemble de données provenant de l'Étude ontarienne de l'incidence des mauvais traitements signalés aux services de protection de l'enfance (ÉOI : Trocmé et al., 1994). Une stratégie d'échantillonnage par strates multiples (multi-stage cluster sampling strategy) a permis d'obtenir un échantillon représentatif de 2447 évaluations de signalements effectués aux agences de protection. Dans un premier temps, 15 sociétés d'aide à l'enfance ont été sélectionnées de manière aléatoire parmi les 51 sociétés affiliées à l'Ontario Association of Chidren's Aid Societies (Trocmé et al., 1994). Dans une seconde étape, 2447 évaluations portant sur des enfants soupçonnés d'avoir été maltraités ont été sélectionnées de manière systématique à partir des évaluations menées entre le $1^{\text {er }}$ mars et le 30 juin 1993 dans les 15 agences. L'analyse qui suit examine les facteurs associés aux décisions d'engager des poursuites pénales dans les cas d'abus physique ${ }^{2}$ $(\mathrm{N}=1003)$ et d'abus sexuel $(\mathrm{N}=577)$. Les évaluations concernant les cas de négligence $(\mathrm{N}=736)$ et de mauvais traitements émotionnels $(\mathrm{N}=131)$ n'ont pas été retenues dans le cadre de ces analyses en raison d'un trop faible pourcentage de cas ayant mené à des poursuites pénales. Ce sous-échantillon de 1580 cas représente plus de 30000 enfants ontariens ayant fait l'objet d'une évaluation en 1993 en raison d'abus physique ou d'abus sexuel signalés, soit un taux d'incidence de plus de 14 enfants pour 1000 .

\section{Procédure}

Un questionnaire de deux pages a été complété par l'intervenant de l'agence de protection responsable de mener l'évaluation du signalement. En moyenne, le questionnaire a été complété environ un mois et demi après la réception du signalement. Le questionnaire documentait les caractéristiques démographiques de la famille faisant l'objet de l'évaluation, une courte liste de facteurs associés, la source du signalement, les caractéristiques des mauvais traitements signalés, le degré de corroboration des faits signalés, les caractéristiques de l'agresseur et les

2. Les types de mauvais traitements sur lesquels on a enquêté ont été classifiés en fonction d'une forme de mauvais traitements primaires et jusqu'à trois autres formes de mauvais traitements secondaires. La présente étude se limite à une analyse des types de mauvais traitements primaires. 
résultats de l'évaluation. L'instrument est une adaptation du questionnaire utilisé pour les études nationales américaines de l'incidence des mauvais traitements (Sedlak, 1991). La validité et la fiabilité de l'instrument ont été examinées dans le cadre d'une étude de dossiers tirés d'évaluations de l'ÉOI (Trocmé et al., 1995a).

\section{Analyse statistique}

Les analyses des situations d'abus physique ou d'abus sexuel ont été faites séparément, compte tenu des différences pouvant exister entre ces deux formes de mauvais traitements concernant les décisions d'entamer des poursuites criminelles. Dans un premier temps, toutes les variables ont été analysées de façon univariée afin de déterminer les variables associées aux poursuites criminelles. Dans une seconde étape, des modèles multivariés ont été construits en utilisant les variables qui s'étaient avérées associées (seuil de signification de $\mathrm{p}<0,05$ ) à la décision d'entamer des poursuites criminelles lors de la première étape. Les analyses multivariées ont été faites en utilisant la procédure de régression logistique du logiciel SPSS (SPSS/PC, version 7.5, 1997). Des analyses de corrélations partielles ont été utilisées pour examiner les risques de colinéarité. Les variables indépendantes ont été introduites en un seul bloc, c'est-à-dire simultanément.

\section{Résultats}

Description des signalements pour abus physique ou abus sexuel

Au total, 577 signalements pour abus sexuel ont fait l'objet d'une évaluation par les services de protection de l'enfance et dans $15 \%$ de ces situations un recours judiciaire a été entrepris contre l'agresseur présumé (tableau 1). Les 577 situations d'abus sexuel se caractérisent par le fait que : 1) plus de la moitié des situations signalées concerne des attouchements sexuels (56\%); 2) lors de l'évaluation, l'intervenant n'a identifié aucune séquelle dans $71 \%$ des situations évaluées; 3 ) un peu moins de la moitié des abus sexuels soupçonnés ou fondés se sont produits à une seule reprise $(47 \%)$; 4 ) les principaux agresseurs présumés sont une figure paternelle (34\%), un membre de la famille autre que le parent (24\%) et une connaissance de la famille (23\%); 5) dans $90 \%$ des situations, l'agresseur présumé est de sexe masculin; 6) les victimes présumées 
14 Criminologie, Vol. $33 \mathrm{~N}^{\circ} 2(2000)$

T A B LE A U 1

Caractéristiques des abus sexuels et physiques signalés aux services de protection de l'enfance en fonction des poursuites criminelles entreprises, Ontario, 1993

\begin{tabular}{|c|c|c|c|c|c|c|}
\hline \multirow[b]{3}{*}{ Total } & \multicolumn{3}{|c|}{ Abus sexuels $(\mathrm{N}=577)$} & \multicolumn{3}{|c|}{ Abus physiques $(\mathrm{N}=1003)$} \\
\hline & \multirow{2}{*}{$\begin{array}{c}\begin{array}{c}\text { Enfants } \\
\text { signalés }\end{array} \\
577\end{array}$} & \multicolumn{2}{|c|}{$\begin{array}{l}\text { Poursuites } \\
\text { criminelles }\end{array}$} & \multirow{2}{*}{$\begin{array}{c}\begin{array}{c}\text { Enfants } \\
\text { signalés }\end{array} \\
1003\end{array}$} & \multicolumn{2}{|c|}{$\begin{array}{l}\text { Poursuites } \\
\text { criminelles }\end{array}$} \\
\hline & & $15 \%$ & - & & $5 \%$ & - \\
\hline Nature des abus sexuels signalés & 561 & - & $p<, 001$ & - & - & n.s. \\
\hline Relation sexuelle & 92 & $71 \%$ & - & - & - & - \\
\hline Attouchement sexuel & 316 & $15 \%$ & - & - & - & - \\
\hline $\begin{array}{l}\text { Exhibitionnisme } \\
\text { et autres formes }\end{array}$ & 153 & $6 \%$ & - & - & - & - \\
\hline Nature des abus physiques signalés & - & - & n.s. & 853 & $5 \%$ & n.s. \\
\hline $\begin{array}{l}\text { Abus liés à des problèmes de } \\
\text { discipline }\end{array}$ & - & - & - & 554 & $6 \%$ & - \\
\hline Autres abus physiques & - & - & - & 252 & $4 \%$ & - \\
\hline Nature des séquelles identifiées & 552 & - & $p<, 001$ & 974 & - & $p<, 001$ \\
\hline Aucune séquelle & 390 & $9 \%$ & - & 638 & $1 \%$ & - \\
\hline Physiques (ex. blessures) & 22 & $27 \%$ & - & 255 & $14 \%$ & - \\
\hline Émotionnelles & 140 & $29 \%$ & - & 81 & $6 \%$ & - \\
\hline $\begin{array}{l}\text { Durée des abus } \\
\text { (cas confirmés ou soupçonnés) }\end{array}$ & 299 & - & $p<, 001$ & 588 & - & n.s. \\
\hline Épisode unique & 141 & $16 \%$ & - & 340 & $7 \%$ & - \\
\hline Moins de 6 mois & 68 & $26 \%$ & - & 76 & $4 \%$ & - \\
\hline Plus de 6 mois & 90 & $31 \%$ & - & 172 & $10 \%$ & - \\
\hline Lien victime/agresseur présumé & 542 & - & n.s. & 920 & - & n.s. \\
\hline Père/conjoint & 183 & $16 \%$ & - & 394 & $6 \%$ & - \\
\hline Mère/conjointe & 22 & $9 \%$ & - & 326 & $3 \%$ & - \\
\hline Deux parents & 21 & $19 \%$ & - & 122 & $7 \%$ & - \\
\hline Membre de la famille & 129 & $14 \%$ & - & 44 & $9 \%$ & - \\
\hline Connaissance & 126 & $18 \%$ & - & 32 & $9 \%$ & - \\
\hline Personne inconnue & 61 & $3 \%$ & - & 27 & $0 \%$ & - \\
\hline Sexe de l'agresseur présumé* & 490 & - & $p<, 05$ & 943 & - & n.s. \\
\hline Masculin & 414 & $17 \%$ & - & 455 & $6 \%$ & - \\
\hline Féminin & 49 & $6 \%$ & - & 357 & $4 \%$ & - \\
\hline Deux sexes & 27 & $15 \%$ & - & 131 & $7 \%$ & - \\
\hline Sexe de l'enfant signalé & 558 & - & n.s. & 978 & - & n.s. \\
\hline Masculin & 184 & $17 \%$ & - & 515 & $5 \%$ & - \\
\hline Féminin & 374 & $14 \%$ & - & 463 & $5 \%$ & - \\
\hline Âge de l'enfant signalé & 558 & - & $p<, 001$ & 986 & - & $\mathrm{p}<, 001$ \\
\hline 0 à 4 ans (exclus) & 78 & $3 \%$ & - & 182 & $7 \%$ & - \\
\hline 4 à 8 ans (exclus) & 185 & $8 \%$ & - & 251 & $2 \%$ & - \\
\hline 8 à 12 ans (exclus) & 142 & $17 \%$ & - & 221 & $6 \%$ & - \\
\hline 12 à 16 ans (exclus) & 153 & $29 \%$ & - & 332 & $6 \%$ & - \\
\hline
\end{tabular}

^chi carrés calculés avec les catégories : masculin et deux sexes combinés. 
sont principalement de sexe féminin $(67 \%)$ et elles sont pratiquement réparties également dans les différents groupes d'âges.

Toujours concernant les abus sexuels, le tableau 2 présente un ensemble de variables associées aux évaluations d'abus sexuel : 1) les familles concernées sont surtout des familles monoparentales dont le chef de famille est la mère (34\%) ou les familles naturelles c'est-à-dire composées des deux parents biologiques (34\%); 2) peu de problèmes familiaux ont été identifiés lors de l'évaluation, les deux principaux étant le fait que la famille vit de l'assistance sociale (28 \%) et dans un logement subventionné (17\%); 3) peu de problèmes liés aux parents ont été identifiés, les deux principaux étant la présence de violence conjugale (13\%) et un problème de santé mentale chez le parent (11\%); 4) près de la moitié des enfants $(49 \%)$ avaient déjà été signalés aux services de protection de l'enfance; et finalement, 5) les signalements proviennent principalement de deux sources soit un membre de la famille (33\%) et le personnel du milieu scolaire de l'enfant (18\%).

Les résultats des évaluations sont présentés au tableau 3. Suite à l'évaluation, l'intervenant a conclu que les abus sexuels étaient fondés dans $31 \%$ des situations évaluées, alors qu'ils étaient soupçonnés dans $27 \%$ des situations. Dans la très grande majorité des cas (90\%), aucune procédure au tribunal de la jeunesse n'était envisagée au moment de l'évaluation, de même qu'aucun placement de l'enfant (91\%). Seulement 26 \% des évaluations ont conduit à des services continus pour l'enfant et sa famille alors que dans $22 \%$ des cas l'évaluation a conduit à une référence et dans $52 \%$ aucun service n'a été offert à la famille. Quarante-quatre pour cent $(44 \%)$ des évaluations d'abus sexuel avaient été menées de manière conjointe par la police et les services de protection.

Mille trois (1003) signalements pour abus physique ont fait l'objet d'une évaluation par les services de protection de l'enfance, et dans seulement $5 \%$ de ces situations un recours judiciaire a été entrepris contre l'agresseur présumé. Tel qu'indiqué au tableau 1, les 1003 situations d'abus physique se caractérisent par le fait que : 1 ) près des deux tiers des situations signalées concernent des problèmes de discipline (65\%); 2) lors de l'évaluation, l'intervenant n'a identifié aucune séquelle dans $66 \%$ des situations évaluées; 3 ) un peu plus de la moitié des abus physiques soupçonnés ou fondés $(58 \%)$ se sont produits à une seule reprise; 4) les figures parentales représentent la très grande majorité des agresseurs présumés (92\%), la proportion de figure maternelle ou paternelle étant similaire; 5) dans $62 \%$ des situations l'agresseur présumé est 
T A B LE A U 2

Facteurs associés aux évaluations pour abus sexuel et physique menées par les services de protection de l'enfance en fonction des poursuites criminelles entreprises,

Ontario, 1993.

\begin{tabular}{|c|c|c|c|c|c|c|}
\hline \multirow[b]{3}{*}{ Total } & \multicolumn{3}{|c|}{ Abus sexuels $(\mathrm{N}=577)$} & \multicolumn{3}{|c|}{ Abus physiques $(\mathrm{N}=1003)$} \\
\hline & \multirow{2}{*}{$\begin{array}{c}\begin{array}{c}\text { Enfants } \\
\text { signalés }\end{array} \\
577\end{array}$} & \multicolumn{2}{|c|}{$\begin{array}{l}\text { Poursuites } \\
\text { criminelles }\end{array}$} & \multirow{2}{*}{$\begin{array}{c}\begin{array}{c}\text { Enfants } \\
\text { signalés }\end{array} \\
1003\end{array}$} & \multicolumn{2}{|c|}{$\begin{array}{l}\text { Poursuites } \\
\text { criminelles }\end{array}$} \\
\hline & & $15 \%$ & - & & $5 \%$ & - \\
\hline Structure familiale & 542 & - & n.s. & 958 & - & n.s. \\
\hline Monoparentale (mère) & 186 & $14 \%$ & - & 262 & $4 \%$ & - \\
\hline Monoparentale (père) & 27 & $11 \%$ & - & 56 & $7 \%$ & - \\
\hline Deux parents biologiques & 186 & $18 \%$ & - & 404 & $5 \%$ & - \\
\hline Recomposée & 115 & $14 \%$ & - & 183 & $4 \%$ & - \\
\hline Autre & 28 & $11 \%$ & - & 53 & $13 \%$ & - \\
\hline \multicolumn{7}{|l|}{ Problématique familiale } \\
\hline Logement insalubre & 13 & $8 \%$ & n.s. & 27 & $7 \%$ & n.s. \\
\hline $\begin{array}{l}\text { Déménagement récent } \\
(<6 \text { mois })\end{array}$ & 73 & $12 \%$ & n.s. & 129 & $6 \%$ & n.s. \\
\hline Logement subventionné & 100 & $21 \%$ & n.s. & 154 & $5 \%$ & n.s. \\
\hline Assistance sociale & 164 & $10 \%$ & $p<, 05$ & 289 & $3 \%$ & n.s. \\
\hline \multicolumn{7}{|l|}{ Problématique parentale } \\
\hline Drogue & 28 & $11 \%$ & n.s. & 48 & $6 \%$ & n.s. \\
\hline Alcool & 44 & $9 \%$ & n.s. & 113 & $5 \%$ & n.s. \\
\hline Santé mentale & 61 & $18 \%$ & n.s. & 125 & $4 \%$ & n.s. \\
\hline Violence conjugale & 73 & $11 \%$ & n.s. & 193 & $6 \%$ & n.s. \\
\hline Signalement antérieur & 561 & - & n.s. & 986 & - & n.s. \\
\hline Oui & 276 & $13 \%$ & - & 457 & $4 \%$ & - \\
\hline Non & 285 & $17 \%$ & - & 529 & $6 \%$ & - \\
\hline Source du signalement & 561 & - & $p<, 001$ & 978 & - & $\mathrm{p}<, 001$ \\
\hline Parent/famille & 183 & $16 \%$ & - & 179 & $4 \%$ & - \\
\hline Enfant victime & 18 & $33 \%$ & - & 46 & $4 \%$ & - \\
\hline Connaissance & 50 & $10 \%$ & - & 117 & $1 \%$ & - \\
\hline Milieu scolaire & 102 & $12 \%$ & - & 340 & $5 \%$ & - \\
\hline Milieu policier & 58 & $43 \%$ & - & 64 & $14 \%$ & - \\
\hline Milieu hospitalier & 22 & $14 \%$ & - & 44 & $18 \%$ & - \\
\hline Autre agence de protection & 18 & $6 \%$ & - & 20 & $20 \%$ & - \\
\hline Autre source & 110 & $4 \%$ & - & 168 & $2 \%$ & - \\
\hline Région géographique & 561 & - & $p<, 001$ & 986 & - & $p<, 001$ \\
\hline Région $\mathrm{A}$ & 174 & $19 \%$ & - & 386 & $7 \%$ & - \\
\hline Région $B$ & 165 & $10 \%$ & - & 211 & $5 \%$ & - \\
\hline Région C & 133 & $12 \%$ & - & 252 & $1 \%$ & - \\
\hline Région D & 89 & $20 \%$ & - & 137 & $6 \%$ & - \\
\hline
\end{tabular}


de sexe masculin alors que cette proportion est de $52 \%$ pour le sexe féminin; 6) les garçons sont légèrement plus souvent la victime des abus physiques signalés soit dans $53 \%$ des situations; 7) les jeunes de 12 à 16 ans (34 \%) sont un peu plus souvent signalés pour abus physique comparativement aux autres groupes d'âge.

Les signalements d'abus physique se caractérisent par le fait que : 1) les familles concernées sont surtout des familles naturelles c'est-à-dire composées des deux parents biologiques (42\%), suivies des familles monoparentales dont le chef de famille est la mère $(27 \%) ; 2$ ) le fait que la famille vit de l'assistance sociale $(28 \%)$ et dans un logement subventionné $(17 \%)$ sont les problèmes familiaux les plus souvent identifiés lors de l'évaluation; 3) la présence de violence conjugale (29\%) et un problème de santé mentale chez le parent $(15 \%)$ sont les deux principaux problèmes liés aux parents identifiés; 4) près de la moitié des enfants $(46 \%)$ avaient déjà été signalés aux services de protection de l'enfance; 5) les signalements proviennent principalement de trois sources : soit du personnel du milieu scolaire $(35 \%)$, soit d'un membre de la famille (18\%), soit d'une personne connue (12\%) (tableau 2).

Finalement, une enquête conjointe menée par les policiers et les intervenants des services de protection de l'enfance n'a été réalisée que dans $20 \%$ des situations évaluées pour abus physique (tableau 3). Dans $25 \%$ des situations évaluées, l'intervenant a conclu que les abus physiques étaient fondés, alors qu'ils étaient soupçonnés dans $28 \%$ des situations; et dans la très grande majorité des cas $(89 \%)$, aucune procédure au tribunal de la jeunesse n'était envisagée au moment de l'évaluation, de même qu'aucun placement de l'enfant (86 \%). Seulement $27 \%$ des évaluations ont conduit à des services continus pour l'enfant et sa famille, alors que dans $15 \%$ des cas l'évaluation a conduit à une référence et dans $57 \%$ des cas aucun service n'a été offert à la famille.

\section{Analyses univariées}

La première étape des analyses consistait à identifier, par une analyse univariée, les variables associées au recours à des poursuites criminelles. Pour les situations d'abus sexuel, les tableaux 1 et 2 montrent que huit variables sont significativement associées au recours : 1) la nature de l'abus sexuel, 2) la nature des séquelles, 3) la durée des abus, 4) l'âge de l'enfant signalé, 5) le sexe de l'agresseur présumé, 6) la source du signalement, 7) le fait que la famille de l'enfant signalé vit de l'assistance 
TABLEA U 3

Résultats des évaluations d'abus sexuel et physique signalés aux services de protection de l'enfance en fonction des poursuites criminelles entreprises, Ontario, 1993

\begin{tabular}{|c|c|c|c|c|c|c|}
\hline \multirow[b]{3}{*}{ Total } & \multicolumn{3}{|c|}{ Abus sexuels $(\mathrm{N}=577)$} & \multicolumn{3}{|c|}{ Abus physiques $(\mathrm{N}=1003)$} \\
\hline & \multirow{2}{*}{$\begin{array}{c}\begin{array}{c}\text { Enfants } \\
\text { signalés }\end{array} \\
577\end{array}$} & \multicolumn{2}{|c|}{$\begin{array}{l}\text { Poursuites } \\
\text { criminelles }\end{array}$} & \multirow{2}{*}{$\begin{array}{c}\begin{array}{c}\text { Enfants } \\
\text { signalés }\end{array} \\
1,003\end{array}$} & \multicolumn{2}{|c|}{$\begin{array}{l}\text { Poursuites } \\
\text { criminelles }\end{array}$} \\
\hline & & $15 \%$ & - & & $5 \%$ & - \\
\hline Corroboration des faits signalés & 560 & - & $\mathrm{p}<, 001$ & 985 & - & $p<, 001$ \\
\hline Faits non fondés & 239 & $7 \%$ & - & 454 & $0 \%$ & - \\
\hline Faits soupçonnés & 150 & $3 \%$ & - & 280 & $3 \%$ & - \\
\hline Faits fondés & 171 & $38 \%$ & - & 251 & $15 \%$ & - \\
\hline Tribunal de la jeunesse & 558 & - & $p<, 001$ & 977 & - & $p<, 001$ \\
\hline Procédure en cours & 39 & $28 \%$ & - & 78 & $23 \%$ & - \\
\hline Procédures considérées & 16 & $44 \%$ & - & 25 & $12 \%$ & - \\
\hline Procédure non considérée & 503 & $13 \%$ & - & 874 & $3 \%$ & - \\
\hline Service offert à la famille & 478 & - & $p<, 001$ & 858 & - & $p<, 001$ \\
\hline Services continus offerts & 124 & $28 \%$ & - & 235 & $17 \%$ & - \\
\hline Services terminés et référés & 104 & $17 \%$ & - & 132 & $4 \%$ & - \\
\hline Services terminés & 250 & $10 \%$ & - & 491 & $1 \%$ & - \\
\hline Placement de l'enfant signalé & 551 & - & $p<, 01$ & 978 & - & $p<, 001$ \\
\hline $\begin{array}{l}\text { Aucun placement n'est } \\
\text { considéré }\end{array}$ & 501 & $13 \%$ & - & 843 & $2 \%$ & - \\
\hline Risque de placement & 20 & $30 \%$ & - & 34 & $26 \%$ & - \\
\hline Placement informel & 8 & $50 \%$ & - & 47 & $13 \%$ & - \\
\hline Placement officiel & 22 & $23 \%$ & - & 54 & $22 \%$ & - \\
\hline $\begin{array}{l}\text { Enquête conjointe } \\
\text { avec policiers }\end{array}$ & 561 & - & $p<, 001$ & 986 & - & $p<, 001$ \\
\hline Oui & 247 & $30 \%$ & - & 197 & $23 \%$ & - \\
\hline Non & 314 & $4 \%$ & - & 789 & $1 \%$ & - \\
\hline
\end{tabular}

sociale, 8) la région géographique où se trouvent les services de protection. À l'exception de la durée, ces variables ont été retenues dans les analyses de régression logistique visant à vérifier les caractéristiques des situations d'abus sexuel dans lesquelles un recours à des poursuites criminelles est entrepris. La durée de l'abus n'a pas été introduit dans le modèle parce que cette variable s'applique seulement aux cas confirmés ou suspectés.

Pour les situations d'abus physique, les tableaux 1 et 2 montrent que quatre variables sont significativement associées au recours à des poursuites criminelles : 1) la nature des séquelles, 2) l'âge de l'enfant signalé, 3) la région géographique où se trouvent les services de protection, 4) la 
source du signalement. Ces variables ont donc été retenues dans les analyses de régression logistique visant à vérifier les caractéristiques des situations d'abus physique dans lesquelles un recours à des poursuites criminelles est entrepris.

Les résultats des évaluations (corroboration, offre de services continus, recours au tribunal, placement, et enquête policière) présentés dans le tableau 3 sont tous significativement associés aux poursuites criminelles, autant pour les évaluations d'abus sexuel que pour les évaluations d'abus physique. Ces variables n'ont pas été introduites dans l'analyse multivariée, puisque les décisions prises par les services de protection sont étroitement liées aux décisions prises par la police et ne peuvent pas être traitées comme des variables indépendantes.

\section{Analyses multivariées}

Parmi les sept facteurs introduits dans le modèle de régression logistique examinant les facteurs associés aux décisions d'entamer des poursuites pénales dans les cas d'abus sexuel, quatre d'entre eux se sont avérés significatifs : la nature des abus sexuels, la nature des séquelles, l'âge de l'enfant signalé et la source de signalement (tableau 4 : Chi carré du modèle $=112,12$ degrés de liberté, $p<0,001)$. La source de signalement est la variable la plus importante $(\mathrm{R}=0,26)$, les cas signalés par la police ou par les enfants ayant un rapport de cote de 10,08 et de 2,18 respectivement. Les abus impliquant des relations sexuelles (Rapport de cote $=13,49$ ) ou des attouchements (Rapport de cote $=4,19)$, ainsi que des abus menant à des séquelles physiques (Rapport de cote $=6,36$ ) ou émotionnelles (Rapport de cote $=2,9$ ) sont également associés aux poursuites criminelles. La probabilité de poursuites augmente en fonction de l'âge de l'enfant signalé (Rapport de cote $=1,15$ par année).

Trois facteurs se sont avérés avoir un effet significatif sur les décisions d'engager des poursuites pénales à la suite d'évaluations d'abus physique (tableau 5) : la présence de blessures, la source de signalement et la région de la province où s'est déroulée l'enquête ${ }^{3}$ (Chi carré du modèle $=90,7$ degrés de liberté, $p<0,001)$. Les séquelles identifiées sont les variables les plus importantes $(R=0,27)$, la présence de blessures physiques et de séquelles émotionnelles ayant des rapports de cote de 9,35 et de 4,69 respectivement. Les signalements provenant de la police, des hôpitaux ou des sociétés d'aide à l'enfance (Rapport de

3. Pour assurer l'anonymat des agences, les zones ne peuvent être identifiées. 
T A B LE A U 4

Régressions logistiques des variables prédisant les poursuites criminelles dans des cas d'abus sexuel, Ontario, 1993

\begin{tabular}{|c|c|c|c|c|c|c|c|}
\hline & B & S.E. & Wald & $\begin{array}{l}\text { degrés } \\
\text { de } \\
\text { liberté }\end{array}$ & Sig & $\mathbf{R}$ & $\begin{array}{l}\text { Rapport de } \\
\text { cote } \\
\text { (Exp B) }\end{array}$ \\
\hline \multicolumn{8}{|l|}{$\begin{array}{l}\text { Nature des abus } \\
\text { sexuels signalés }\end{array}$} \\
\hline $\begin{array}{l}\text { Exhibitionnisme } \\
\text { et autres formes } \\
\text { (contraste) }\end{array}$ & - & - & 21,6 & 2 & 0,000 & 0,203 & - \\
\hline Attouchement sexuel & 1,433 & 0,538 & 7,1 & 1 & 0,008 & 0,109 & 4,19 \\
\hline Relation sexuelle & 2,602 & 0,594 & 19,2 & 1 & 0,000 & 0,201 & 13,49 \\
\hline \multicolumn{8}{|l|}{$\begin{array}{l}\text { Nature des séquelles } \\
\text { identifiées }\end{array}$} \\
\hline $\begin{array}{l}\text { Aucune séquelle } \\
\text { (contraste) }\end{array}$ & - & - & 15,1 & 2 & 0,001 & 0,161 & - \\
\hline $\begin{array}{l}\text { Physiques (ex. } \\
\text { blessures) }\end{array}$ & 1,8494 & 0,666 & 7,7 & 1 & 0,006 & 0,115 & 6,36 \\
\hline Émotionnelles & 1,0651 & 0,324 & 10,8 & 1 & 0,001 & 0,143 & 2,90 \\
\hline Âge de l'enfant signalé & 0,144 & 0,042 & 12,0 & 1 & 0,001 & 0,153 & 1,15 \\
\hline \multicolumn{8}{|l|}{ Source du signalement } \\
\hline autre source (contraste) & - & - & 33,0 & 2 & 0,000 & 0,260 & - \\
\hline enfant & 0,778 & 0,689 & 1,3 & 1 & 0,259 & 0,000 & 2,18 \\
\hline milieu policier & 2,311 & 0,405 & 32,6 & 1 & 0 & 0,268 & 10,08 \\
\hline $\begin{array}{l}\text { Un des agresseurs } \\
\text { est de sexe masculin }\end{array}$ & 0,715 & 0,771 & 0,9 & 1 & 0,354 & 0,000 & 2,04 \\
\hline Assistance sociale & $-0,529$ & 0,357 & 2,2 & 1 & 0,139 & $-0,021$ & 0,59 \\
\hline \multicolumn{8}{|l|}{ Région géographique } \\
\hline Région D (contraste) & - & - & 6,8 & 3 & 0,078 & 0,044 & - \\
\hline Région A & 0,357 & 0,413 & 0,7 & 1 & 0,388 & 0,000 & 1,43 \\
\hline Région $B$ & $-0,732$ & 0,469 & 2,4 & 1 & 0,118 & $-0,032$ & 0,48 \\
\hline Région C & $-0,079$ & 0,458 & 0,0 & 1 & 0,863 & 0,000 & 0,92 \\
\hline Constante & $-5,832$ & 1,036 & 31,7 & 1 & 0 & - & - \\
\hline Chi carré du modèle & 112 & - & - & 12 & 0,000 & - & - \\
\hline
\end{tabular}


TABLEA U 5

Régressions logistiques des variables prédisant les poursuites criminelles dans des cas d'abus physique, Ontario, 1993

\begin{tabular}{|l|c|c|c|c|c|c|c|}
\hline & B & S.E. & Wald & $\begin{array}{c}\text { degrés } \\
\text { de } \\
\text { liberté }\end{array}$ & Sig & R & $\begin{array}{c}\text { Rapport } \\
\text { de cote } \\
\text { (Exp B) }\end{array}$ \\
\hline $\begin{array}{l}\text { Nature des } \\
\text { séquelles identifiées }\end{array}$ & & & & & & & \\
\hline Aucune séquelle (contraste) & - & - & 32,4 & 2 & 0,000 & 0,268 & - \\
\hline Physiques (ex. blessures) & 2,236 & 0,393 & 32,4 & 1 & 0,000 & 0,278 & 9,35 \\
\hline Émotionnelles & 1,545 & 0,584 & 7 & 1 & 0,008 & 0,1128 & 4,69 \\
\hline $\begin{array}{l}\text { Enfant signalé entre } \\
\text { 4 et 8 ans (exclus) }\end{array}$ & $-0,858$ & 0,499 & 2,9 & 1 & 0,085 & $-0,049$ & 0,42 \\
\hline $\begin{array}{l}\text { Signalement venant du } \\
\text { milieu hospitalier, policier } \\
\text { ou d'une autre agence } \\
\text { de protection }\end{array}$ & 1,367 & 0,337 & 16,5 & 1 & 0,000 & 0,191 & 3,92 \\
\hline Région géographique & & & & & & & \\
\hline Région C (contraste) & - & - & 10,8 & 3 & 0,012 & 0,111 & - \\
\hline Région A & 1,792 & 0,629 & 8,1 & 1 & 0,004 & 0,125 & 6,00 \\
\hline Région B & 0,942 & 0,685 & 1,9 & 1 & 0,169 & 0 & 2,57 \\
\hline Région D & 1,504 & 0,710 & 4,4 & 1 & 0,034 & 0,079 & 4,50 \\
\hline Constante & $-5,627$ & 0,670 & 70,6 & 1 & 0,000 & - & - \\
\hline Chi carré du modèle & - & - & 7 & 0,000 & - & - \\
\hline
\end{tabular}

cote $=3,92$ ), et la région géographique ou s'est déroulée l'enquête (Rapport de cote de la région $\mathrm{A}$ en contraste avec la région $\mathrm{C}=6,00$ ) se maintiennent également de manière significative dans le modèle.

\section{Discussion}

L'Étude ontarienne sur l'incidence des mauvais traitements est une des premières études examinant en détails les caractéristiques des situations signalées aux sociétés de protection de l'enfance. L'analyse présentée cidessus examine les évaluations menées conjointement avec la police et donne un aperçu descriptif du rôle joué par les protocoles d'intervention élaborés conjointement par les sociétés de protection d'enfants et les services policiers. 
Niveau de collaboration entre les services de protection de l'enfance et les services policiers

Près d'un tiers des situations d'abus physique ou d'abus sexuel documentées par l'ÉOI impliquent également une enquête policière, et dans $6,6 \%$ des situations évaluées ou dans $30 \%$ des situations impliquant également une enquête policière des poursuites criminelles ont été entamées, ce qui constitue un pourcentage élevé en comparaison du $4 \%$ évalué par Tjaden et Thoennes (1992) en Californie. Les décisions prises par les services de protection (par exemple, la décision de placer l'enfant) dans le cadre de l'ÉOI étaient clairement associées aux décisions d'entamer des poursuites criminelles. Une augmentation significative des taux de criminalisation est associée aux décisions de confirmer la présence d'abus sexuel ou physique, de fournir des services de protection continus, de faire intervenir le tribunal de la jeunesse et de placer les enfants en foyer d'accueil (voir tableau 3). Alors que cette concordance n'est pas surprenante, il faut néanmoins noter que $84 \%$ des cas d'abus physique et $62 \%$ des cas d'abus sexuel confirmés par les sociétés de protection n'ont pas mené à des poursuites criminelles.

Bien que nous n'ayons pas de données québécoises à ce sujet, une étude effectuée par l'Association des centres jeunesse du Québec sur la pratique des intervenants concernant l'application du protocole socio-judiciaire au sein de toutes les équipes RTS et É/O du Québec, montrait qu'il existe un écart entre la perception des intervenants qui disent favoriser et entériner, en grande majorité, la concertation socio-judiciaire dans les cas d'abus sexuel envers les enfants et la mise en application des critères de divulgation policière qui permet cette concertation (Boucher, 1995). Boucher concluait que la concertation socio-judiciaire est appliquée sporadiquement, et elle semble dépendre des intervenants en place. Selon un document de la Division des crimes majeurs, portant sur l'analyse des abus sexuels survenus sur le territoire de la Communauté urbaine de Montréal en 1996 (SPCUM, 1997), 57 \% des 1383 plaintes policières concernaient un enfant de moins de 18 ans. De ces 791 plaintes, 88 \% n'impliquaient pas l'usage d'un protocole avec une autre organisation.

De plus, certains résultats de recherches suggèrent que les perceptions qu'entretiennent les intervenants des services de protection, et de façon plus générale les professionnels des services sociaux, quant à l'impact négatif que peut avoir le système judiciaire sur l'enfant, pourraient être un des éléments ne favorisant pas la collaboration entre les deux systèmes (Tedesco et Schnell, 1987; Tidwell et al., 1990 : voir Lipovsky, 1994). 
La différence entre les taux de poursuites criminelles pour les cas d'abus physique et les taux de poursuites pour abus sexuel est notable. La police était impliquée dans $20 \%$ des évaluations pour abus physique et dans $43 \%$ des évaluations pour abus sexuel; des poursuites pénales furent engagées dans $5 \%$ des évaluations pour abus physique - soit $25 \%$ des évaluations conjointes -, et dans $15 \%$ des évaluations pour abus sexuel - soit $34 \%$ des évaluations conjointes (voir tableau 3). Tjaden et Thoennes (1992) ont également trouvé qu'en Californie le système criminel était beaucoup plus souvent utilisé dans le cas d'abus sexuel. Le taux relativement bas de poursuites criminelles dans les cas d'abus physique est peut-être une indication de l'ambiguiité, chez les professionnels et le public en général, concernant l'utilisation de la force physique comme pratique éducative envers les enfants (Durrant, 19931994; Durrant et Rose-Crasnor, 1995; McCord, 1995).

Facteurs associés au recours

à des poursuites criminelles

Les analyses multivariées confirment cette différence d'approche dans les cas d'abus sexuel par rapport aux cas d'abus physique. Dans les cas d'abus sexuel, la sévérité de l'acte est fortement associée aux poursuites pénales, les cas de rapports sexuels ayant la plus grande probabilité de mener à des poursuites, tandis que les cas où il n'y a aucun contact mènent bien plus rarement à des poursuites. Dans les cas d'abus physique, c'est la présence de blessures qui accrô̂t la probabilité de mener à des poursuites. Tjaden et Thoennes (1992) ont noté une même relation entre la présence de blessures et la décision d'entamer des poursuites pénales; malheureusement leurs analyses n'ont pas comparé les différentes formes d'abus.

L'âge de l'enfant apparaît également jouer un rôle crucial dans les décisions prises dans les cas d'abus sexuel. Les poursuites pénales sont significativement plus probables dans les cas où les victimes sont plus âgées. L'influence du facteur âge peut s'expliquer en fonction de la capacité de l'enfant de témoigner. Tjaden et Thoennes (1992) ont également noté une même relation entre l'âge de la victime et la décision d'entamer des poursuites pénales. Une différence notable, cependant, est que dans l'étude de Tjaden et Thoennes (1992), l'effet de l'âge est inversé : les poursuites pénales sont moins fréquentes lorsque les victimes sont adolescentes. Les auteurs notent qu'aux États-Unis le comportement sexuel de victimes adolescentes peut affecter la crédibilité de leur témoignage. 
L'étude a également démontré qu'un certain nombre de facteurs extrinsèques semblaient avoir une influence sur les décisions de procéder à des poursuites criminelles. La source de signalement joue un rôle déterminant dans les deux types d'évaluations. Ce résultat n'est pas surprenant étant donné que des études ont révélé que les sources de signalement sont associées à un certain nombre de décisions d'intervention (Groenveld et Giovannoni, 1977; Runyan et al., 1981; Miller et al., 1982; Giovannoni, 1989; Lindsey, 1991). Il est néanmoins important d'interpréter ces données avec une certaine circonspection. Bien que certains concluent que cette association indique que la source du signalement a une influence excessive sur les résultats d'évaluations (Groenveld et Giovannoni, 1977; Runyan et al., 1981; Lindsey, 1991), il est possible que cette association apparente soit confondue par le fait que certaines sources sont en contact avec des situations qui en elles-mêmes sont plus graves et méritent donc une intervention plus ciblée (Giovannoni, 1989; Tjaden et Thoennes, 1992; Winefield et Bradley, 1992; Trocmé et al., 1995b). L'analyse présentée ici démontre que l'impact de la source du signalement est en partie modéré par des facteurs intrinsèques aux mauvais traitements, tels que la sévérité, le type de mauvais traitement et l'âge de l'enfant, mais continue néanmoins à jouer un rôle indépendant important.

Pour les abus physiques, les cas signalés par la police, par les hôpitaux et par les autres agences de protection ont significativement plus de probabilités de mener à des poursuites judiciaires. Les taux de poursuites pénales élevés pour les cas signalés par les hôpitaux reflètent le fait que ces derniers sont en contact avec des enfants blessés et sont donc exposés à des cas plus sérieux. Par la nature même de leur fonction, la police et les agences de protection sont exposées à des cas plus sérieux ${ }^{4}$ et sont en meilleure position pour identifier parmi ces cas ceux qui nécessitent une intervention. En effet, dans leur étude des standards définitionnels utilisés par différentes professions, Giovannoni et Becerra (1979) ont trouvé que les professionnels qui ont un contact régulier avec les familles qui maltraitent leurs enfants ont tendance à ne signaler que les cas les plus sérieux.

Dans le cas d'abus sexuel, ce sont les signalements faits par les enfants eux-mêmes ou par la police qui ont une plus grande probabilité de mener à des poursuites judiciaires. Le taux plus élevé de poursuites dans

4. Alors que notre étude contrôle en partie la sévérité en fonction de la nature des abus et des séquelles, les mesures catégorielles utilisées ne sont pas assez fines pour tenir compte complètement des différences circonstancielles qui affectent l'évaluation de la sévérité. 
les cas signalés par les enfants souligne l'importance du témoignage de la victime. Cette relation montre que, si un cas est signalé par un enfant, ceci indique que celui-ci est probablement en meilleure position pour témoigner.

Un phénomène plus difficile à expliquer est l'influence des facteurs géographiques dans le cadre de poursuites criminelles pour abus physique. Ce même phénomène a été noté dans une étude des facteurs associés aux décisions de placer les enfants en foyers d'accueil (Runyan et al., 1981). L'étude de Tjaden et Thoennes (1992) montre également que la proportion de cas menant à des poursuites pénales varie géographiquement, mais cette variation ne s'est pas avérée statistiquement significative. Un examen du profil démographique des quatre régions n'indique pas de raison apparente pouvant suggérer que les régions où les poursuites criminelles sont plus probables auraient à enquêter sur des situations plus sérieuses de mauvais traitements. Il semblerait donc que les standards utilisés par la police et les agences de protection varient d'une région à l'autre.

Un certain nombre de facteurs secondaires qui avaient été identifiés dans des études précédentes ne se sont pas avérés associés aux décisions d'entamer des poursuites criminelles. Tjaden et Thoennes (1992) avaient trouvé que la race de l'accusé était liée à l'intervention du système criminel. Certains ont remarqué que la race et la classe sociale étaient liées à d'autres décisions prises par les intervenants des agences de protection de l'enfance (Runyan et al., 1981; Hampton, 1987; Lindsey, 1991). Ni la pauvreté ni la race de l'accusé ne sont associées aux poursuites criminelles dans la présente étude. Il est également intéressant de noter que les facteurs de risque examinés - la toxicomanie, les problèmes de santé mentale et la violence conjugale - n'apparaissent pas affecter les décisions d'entreprendre des poursuites pénales. Il est particulièrement intéressant de noter que l'analyse multivariée montre que les poursuites ne sont pas un élément déterminant dans les cas où il y a eu un divorce ou une séparation, étant donné que certains maintiennent que les mères sont coupables de fausses allégations d'abus dans les cas de divorces contestés (Faller, 1995).

Alors que Tjaden et Thoennes (1992) avaient trouvé que les poursuites criminelles étaient beaucoup plus rares quand l'accusé était un parent, la présente étude a trouvé que la relation de l'accusé avec l'enfant n'est pas associée aux décisions d'entamer des poursuites criminelles. Contrairement à l'impression selon laquelle il est plus difficile d'intervenir dans des situations intra-familiales, les données de l'ÉOI indiquent 
que la police entreprend des poursuites criminelles, quelle que soit la relation entre l'accusé et la victime.

\section{Aspects méthodologiques}

L'ÉOI représente un effort intéressant dans le développement d'une compréhension de la relation entre les services de protection et les services policiers quant à la décision d'entreprendre des poursuites criminelles dans les situations d'abus physique ou d'abus sexuel. Le nombre de situations documentées et le nombre de variables qui ont été considérées pour comprendre ce phénomène sont deux aspects méthodologiques forts de l'étude. Il est néanmoins important de souligner certaines limites de cette banque de données.

L'ÉOI est une étude transversale dont les données ont été recueillies, en moyenne, un mois après la réception du signalement aux services de protection. La nature transversale du devis fait en sorte que pour certaines variables, les informations obtenues au cours de l'étude doivent être considérées comme partielles. Par exemple, il est possible que, dans certaines situations, des plaintes policières ou des poursuites pénales aient été entreprises après la fin de la collecte de données. Par conséquent, il faut s'attendre à une certaine sous-estimation des taux de poursuites criminelles dans la présente étude. De même, il est probable que les mauvais traitements dévoilés lors du signalement puissent être précisés davantage en cours d'intervention. Les recherches indiquent que, pour l'enfant, le dévoilement d'un abus sexuel est un processus particulièrement complexe : il consiste parfois en un mélange de dénégations, de révélations complètes ou partielles et de rétractations de la part de la victime (Sorensen et Snow, 1991; Gonzalez et al., 1993; Elliott et Brière, 1994). De plus, l'expérience clinique et les recherches montrent que le dévoilement doit davantage être examiné comme un processus plutôt qu'un événement : la volonté de dévoiler l'abus et de préciser la nature exacte de cet abus augmente généralement avec le temps (Sorensen et Snow, 1991).

Malgré le fait que la présente étude ait examiné le rôle de plusieurs variables dans la décision d'entreprendre des poursuites criminelles, certains facteurs n'ont pas été considérés. Trois facteurs apparaissent particulièrement importants à considérer dans les recherches futures, soit : 1) la qualité et la nature des preuves et témoignages mis à la disposition de la police; 2) la perception qu'ont les principaux acteurs (l'enfant, le parent non agresseur et l'intervenant des services de protection) impliqués dans la situation de mauvais traitements concernant le système judiciaire et son 
impact sur l'enfant victime; 3) le niveau de fonctionnement psychosocial de l'enfant signalé. Par exemple, alors que la probabilité de poursuites criminelles est significativement augmentée par le fait qu'une enquête est confirmée par les intervenants de la protection, la majorité des cas confirmés n'a pas donné lieu à de telles poursuites. Cette différence s'explique en partie par le fait que le niveau de preuve nécessaire pour justifier des poursuites criminelles est plus élevé que le niveau de preuve nécessaire à un intervenant d'une agence de protection pour déterminer qu'un enfant a probablement été maltraité. Le fait que les poursuites soient plus fréquentes dans les cas impliquant des victimes plus âgées suggère que la qualité des preuves joue un rôle important.

Certaines études ont également montré qu'il y avait des proportions importantes de parents, d'enfants maltraités et d'intervenants des services de protection qui pensent que le témoignage de l'enfant à la cour peut avoir des conséquences négatives pour ce dernier (Tedesco et Schnell, 1987; Tidwell et al., 1990 : voir Lipovsky, 1994; Oates et al., 1995). Ces perceptions peuvent donc avoir un effet dissuasif dans la décision d'entreprendre des recours judiciaires contre l'agresseur.

\section{Conclusion}

Étant donné le développement de protocoles d'entente entre les services policiers et ceux de la protection de l'enfance, et le nombre croissant de cas qui mènent à des poursuites criminelles, il demeure important de mieux comprendre la nature de cette collaboration et les facteurs qui influencent le recours aux poursuites criminelles. Le fait que les deux tiers des situations évaluées par les services de protection ne mènent pas à des poursuites pénales indique que ces deux systèmes d'intervention collaborent encore peu et doivent continuer à élaborer des procédures qui peuvent assurer une approche conjointe plus efficace.

Cette étude offre un premier aperçu de certains facteurs qui influencent les décisions d'engager des poursuites pénales à la suite d'évaluations de cas d'abus physique ou d'abus sexuel; des études plus détaillées sont toutefois requises pour comprendre comment les décisions sont prises. Les deux modèles élaborés ci-dessus ne tiennent compte que d'une partie des facteurs concernés, et ne tiennent pas suffisamment compte de la qualité des preuves mises à la disposition des enquêteurs, du contexte des mauvais traitements, des perceptions des différents acteurs (parents, enfants, intervenants, policiers, avocats et juges) impli- 
qués dans la collaboration et de l'état psychologique des enfants. De plus, il serait important d'enrichir ce type d'analyse quantitative avec des données qualitatives qui examineraient en profondeur les processus qui mènent à de telles décisions. En l'absence de telles analyses, il est impossible de commencer à évaluer l'impact des nouvelles lois fédérales sur les mauvais traitements ni l'impact des protocoles d'interventions qui ont été mis sur pied pour assurer une meilleure collaboration entre les services de protection de l'enfance et les services policiers.

\section{Références}

BARSKY, A. et TrocmÉ, N. 1997. « The Essential Aspects of Mediation in Child Protection Cases » Children and Youth Services Review 19 (3) : 1-18.

Besharov, D. 1990. « Gaining Control over Child Abuse Reports » Public Welfare $48(2): 34-40$.

BOUCHER, L. 1995. Bilan sur les pratiques des centres jeunesse du Québec concernant l'application de l'approche socio-judiciaire dans le traitement des abus sexuels envers les enfants, Montréal : Association des Centres jeunesse du Québec.

Burford, G. et Pennell, J. 1995. «Family Group Decision Making: An Innovation in Child and Family Welfare », Pp. 140-153 in Child Welfare in Canada: Research and Policy Implications, sous la direction de B. Galaway et J. Hudson. Toronto : Thompson Educational Press.

Cross, T.P., De Vos, E. et Whitcomb, D. 1994. « Prosecution of Child Sexual Abuse: Which Cases Are Accepted? » Child Abuse and Neglect 18 (8) : 663-677.

DURRANT, J.E. 1993-1994. « Sparing the Rod: Manitobans' Attitudes Toward the Abolition of Physical Discipline and Implications for Policy Change » Canada's Mental Health Winter : 2-6.

Durrant, J.E. et ROSE-CraSNOR, L. 1995. Corporal Punishment: Research Review and Policy Recommendations, Ottawa: Health and Welfare Canada, Family Violence Prevention Division.

ElliotT, D.M. et BRIERE, J. 1994. « Forensic Sexual Abuse Evaluations of Older Children: Disclosures and Symptomatology » Behavioral Sciences and the Law 12 (3) : 261-277.

FALLER, K.C. 1995. "Allegations of Sexual Abuse in Divorce " Journal of Child Sexual Abuse 4 (4) : 1-25.

GiovanNONI, J.M. 1989. « Substantiated and Unsubstantiated Reports of Child Maltreatment » Children and Youth Services Review 11 : 299-318.

Giovannoni, J.M. et BeCerra, R.M. 1979. Defining Child Abuse, New York : Free Press.

Gonzalez, L.S., Waterman, J., Kelly, R.J., McCord, J. et Oliveri, M.K. 1993. "Children's Patterns of Disclosures and Recantations of Sexual and Ritualistic Abuse Allegations in Psychotherapy "Child Abuse and Neglect 17 (1) : 81-89. 
Gray, E. 1993. Unequal Justice: The Prosecution of Child Sexual Abuse, New York : Free Press.

Groenveld, L.P. et Giovannoni, J. M. 1977. « Disposition of Child Abuse and Neglect Cases » Social Work Research שV Abstract 13 : 24-30.

HAMPTON, R.L. 1987. "Race, Class and Child Maltreatment » Journal of Comparative Family Studies 18 (1) : 113-226.

LANNING, K. 1996. "Criminal Investigation of Sexual Victimization of Children », Pp. 247-263 in The APSAC Handbook on Child Maltreatment, sous la direction de J. Brière, L. Berliner, J. Bulkley, J. Carole et T. Reid. Thousand Oaks : Sage.

LINDSEY, D. 1991. «Factors Affecting the Foster Care Placement Decision: An Analysis of National Survey Data » American Journal of Orthopsychiatry 61 (2) : 272-281.

LINDSEY, D. 1994. The Welfare of Children, London : Oxford University Press.

LiNDSEY, D. et REGEHR, C. 1993. "Protecting Severely Abused Children: Clarifying the Roles of Criminal Justice and Child Welfare » American Journal of Orthopsychiatry 63 : 509.

LIPOVSKY, J.A. 1994. « The Impacts of Court on Children: Research Findings and Practical Recommendations » Journal of Interpersonal Violence 9 (2) : 237-257.

MCCORD, J. 1995. Coercion and Punishment in Long-term Perspectives, Cambridge : Cambridge University Press.

Miller, B., Shireman, J., Burke, P. et Brown, F. 1982. « System Responses to Initial Reports of Child Abuse and Neglect Cases " Journal of Social Service Research 5 (3/4) : 95-111.

OAtes, R.K., Lynch, D.L., Stern, A.E., O’ToOle, B.I. et CoOney, G. 1995. "The Criminal Justice System and the Sexually Abused Child: Help or Hindrance? » Medical Journal of Australia 162 (3) : 126-130.

Ontario, 1990. Lois sur les services à l'enfance et à la famille. Lois refondues de l'Ontario de 1990, Chapitre C. 11. Toronto : Imprimeur de la reine pour l'Ontario.

PALMER, S. 1989. « Mediation in Child Protection Cases: An Alternative to the Adversary System » Child Welfare 68 (1) : 21-31.

ROBERGE, P. 1998. Les parcours de six cohortes: enfants et adolescents signalés à la direction de la protection de la jeunesse, Québec : Ministère de la Santé et des Services sociaux, Direction de la recherche et de l'évaluation.

RunYan, D.K., Gould, C.L., Trost, D. et LADA, F.A. 1981. « Determinants of Foster Care Placement for the Maltreated Child » American Journal of Public Health 71 (7) : 706-710.

Sas, L.D., Austin, G., Wolfe, D.A. et Hurley, P. 1991. Reducing the Systeminduced Trauma for Child Sexual Abuse Victims Throught Court Preparations, Assessment, and Follow-up, Ottawa : National Welfare Grants Division, Health and Welfare Canada (Project \#4555-1-125).

SCHMOLKA, V. 1992. Le projet de loi C-15 donne-t-il les effets voulus?, compte rendu des recherches portant sur les effets des modifications de 1988 relatives à l'exploitation sexuelle des enfants, Ottawa: Ministère de la Justice du Canada. 
SedlaK, A. 1991. National Incidence and Prevalence of Child Abuse and Neglect: 1988, Rockville : Westat.

Sorensen, T. et SnOw, B. 1991. "How Children Tell: The Process of Disclosure in Child Sexual Abuse » Child Welfare LXX (1) : 3-15.

SPCUM 1997. Analyse des agressions sexuelles pour l'année 1996, Montréal: Services Policiers de la Communauté Urbaine de Montréal, Division des crimes majeurs.

Tedesco, J. F. et Schnell, S. V. 1987. "Children's Reactions to Sex Abuse Investigation and Litigation » Child Abuse and Neglect 11 (3) : 267-272.

TJaden, P. G. et Thoennes, N. 1992. «Predictors of Legal Intervention in Child Maltreatment Cases » Child Abuse and Neglect 16 (6) : 807-821.

TOURIGNY, M. 1999. "Facteurs et conséquences psychosociales liés au placement des enfants victimes d'agressions sexuelles intrafamiliales » Revue canadienne de psychoéducation 28 (2) : 225-245.

TrocmÉ, N. 1991. "Child Welfare Services », Pp 63-91 in State of the Child in Ontario, sous la direction de D. Barnhorst et L. Johnson. Toronto : Oxford University Press.

Trocmé, N., McPheE, D., TAM, K.K. et HaY, T. 1994. Ontario Incidence Study of Reported Child Abuse and Neglect, Toronto: Institute for the Prevention of Child Abuse.

Trocmé, N., Michalski, J., McPhee, D., Tam, K.K. et Scarth, S. 1995a. Canadian Incidence Study of Reported Child Maltreatment: Methodology and Feasibility Review, Toronto : Centre for Applied Social Research, University of Toronto, Faculty of Social Work.

TrocmÉ, N., TAM, K.K. et McPheE, D. 1995b. « Correlates of Substantiation of Maltreatment in Child Welfare Investigations », Pp. 20-40 in Child Welfare in Canada: Research and Policy Implications, sous la direction de B. Galaway et J. Hudson. Toronto : Thompson Educational Press.

WALSH, B. 1996. «Criminal Investigation of Physical Abuse and Neglect », Pp. 247-263 in The APSAC Handbook on Child Maltreatment, sous la direction de J. Brière, L. Berliner, J. Bulkley, J. Carole et T. Reid. Thousand Oaks : Sage.

Wells, M. 1990. Canada's Law on Child Sexual Abuse: A Handbook, Ottawa : Department of Justice, Canada.

Whitcomb, D., Runyan, D.K., De Vos, E., Hunter, W.M., Cross, T.P., Everson, M.D., Peeler, N.A., Porter, C.Q. Toth, P.A. et Cropper, C. 1991. Child Victim as Witness Research and Development Program. Final Report. Grant number 87-MC-CX-0026. Office of Juvenile Justice and Delinquency Prevention, Office of Justice Programs, U.S. Department of Justice.

Winefield, H.R. et BRAdley, P.W. 1992. «Substantiation of Reported Child Abuse or Neglect: Predictors and Implications » Child Abuse and Neglect 16 : 661-671.

WolOCK, I. 1982. "Community Characteristics and Staff Judgments in Child Abuse and Neglect Cases » Social Work Research and Abstracts 18 (2) : 9-15. 